BULLETIN Bulletin hispanique

HISPANIQUE Université Michel de Montaigne Bordeaux

116-1 | 2014

Varia

\title{
La politique sanitaire du gouvernement français à l'égard des réfugiés espagnols de la guerre civile
}

\section{Vincent Parello}

\section{(2) OpenEdition}

1 Journals

Édition électronique

URL : http://journals.openedition.org/bulletinhispanique/3046

DOI : 10.4000/bulletinhispanique.3046

ISSN : $1775-3821$

Éditeur

Presses universitaires de Bordeaux

Édition imprimée

Date de publication : 1 juin 2014

Pagination : 247-262

ISBN : 978-2-86781-931-5

ISSN : 0007-4640

Référence électronique

Vincent Parello, « La politique sanitaire du gouvernement français à l'égard des réfugiés espagnols de la guerre civile », Bulletin hispanique [En ligne], 116-1 | 2014, mis en ligne le 01 juin 2017, consulté le 23 mai 2020. URL : http://journals.openedition.org/bulletinhispanique/3046 ; DOI : https://doi.org/ 10.4000/bulletinhispanique.3046 


\title{
La politique sanitaire du gouvernement français à l'égard des réfugiés espagnols de la guerre civile
}

\author{
Vincent Parello \\ Université Bordeaux Montaigne
}

Au nom de la sûreté nationale et du droit d'asile, il incombait à l'État de préserver les populations françaises du risque de contagion, et de secourir et de sauver des êtres humains menacés de mort. Cela dit, la logique de police du gouvernement n'excluait nullement la logique humanitaire qui s'effectuait par le biais de l'aide privée nationale et internationale.

Mots-clés : réfugiés espagnols, guerre civile, politique sanitaire.

En nombre de la seguridad nacional y del derecho de asilo, incumbia al Estado preservar las poblaciones francesas del riesgo de contaminio, y socorrer y salvar seres humanos amenazados de muerte. Sin embargo, la lógica policíaca del gobierno no impedía la lógica humanitaria que se efectuaba mediante la ayuda privada nacional e internacional.

Palabras claves: refugiados españoles, guerra civil, política sanitaria.

In the name of national security and right of asylum, preserving the French population from the risks of contagion was incumbent on the State, as well as rescuing and saving human beings on the verge of death. Yet, the policin logic of the Government did not exclude in the least the humanitarian aid, which was carried out by national and international private assistance.

Keywords: spanish refugees, civil war, health policy.

$\mathrm{P}$

endant toute la durée de la guerre civile, la frontière franco-espagnole demeura, à de rares exceptions près, hermétiquement close. Il y avait à cela des raisons d'ordre politique. En vertu de la déclaration franco-britannique de non-intervention signée le 15 août 1936, la France se devait d'observer la plus stricte neutralité dans le conflit qui déchirait nationalistes et républicains. Dans son fameux discours prononcé à Luna-Park le 6 septembre 1936, Léon Blum, alors président du Conseil, s'expliquait sur les raisons de ce choix. À son avis, il était 
« impossible d'agir autrement sans ouvrir en Europe une crise dont il serait difficile ou dont il serait malheureusement trop facile de prévoir les conséquences ${ }^{1}$. En d'autres termes, l'abandon de la politique de non-intervention impliquait l'intervention, laquelle conduisait inéluctablement à la guerre.

Si la France se refusa à envoyer des canons et des avions pour l'Espagne, en dépit des revendications des communistes et de certains secteurs de la gauche, elle remplit toutefois son devoir d'humanité en soulageant la misère de milliers de réfugiés espagnols qui se pressèrent à ses portes après l'effondrement du front de Catalogne. À l'issue d'une conférence interministérielle qui se tint à Paris le 26 janvier 1939, le gouvernement prit la décision d'ouvrir la frontière aux personnes munies d'autorisations délivrées par les agents consulaires français et à « 3000 enfants espagnols qui grelottaient de froid et de faim $»^{2}$. Le 28 janvier, la France accueillit les civils et les soldats blessés, avant de refermer aussitôt sa frontière. Le 29 janvier, en effet, le ministère de l'Intérieur publiait le communiqué suivant : "La frontière française est fermée aux hommes en état de porter les armes. Par souci d'humanité, on accueille les femmes, les enfants, les vieillards, les malades, mais l'entrée du territoire est interdite aux hommes valides $»^{3}$. Sous la pression des événements, les combattants de l'armée républicaine obtinrent finalement l'autorisation de passer en France par Cerbère le 5 février et par le Perthus le 6 février. À la mi-février, on estime que les réfugiés espagnols étaient au nombre de 453 000, dont 270000 militaires, 170000 civils et 13000 malades et blessés. En y ajoutant ceux qui avaient franchi les Pyrénées en dehors des postes-frontières ou sans avoir été contrôlés par les autorités, on atteint le chiffre de 500000 que la plupart des historiens actuels acceptent comme étant le plus proche de la réalité ${ }^{\text {. }}$

Cela dit, quelles furent les mesures édictées par le ministère de la Santé publique sous le gouvernement radical de Daladier ? Quelles solutions adopta le gouvernement français vis-à-vis de ces 13000 victimes civiles et militaires de la guerre civile espagnole ? Quels enjeux idéologiques se cachaient derrière la politique sanitaire? Autant de questions auxquelles nous essaierons de répondre dans les pages qui suivent.

\section{LA POlitique SANITAIRE DU GOUVERNEMENT FRANÇAIS VIS-À-VIS DES ÉTRANGERS ET DES RÉFUGIÉS}

Avec l'arrivée au pouvoir des radicaux au printemps 1938, le gouvernement Daladier promulgua toute une série de lois visant à réglementer, dans un sens plus restrictif, la présence des étrangers sur le territoire national. C'est ainsi

1. L'œuvre de Léon Blum, Paris, Albin Michel, 1964, p. 393. Cette œuvre a été publiée sans nom d'auteur. Le texte sténographique du discours prononcé à la grande manifestation socialiste de Luna-Park est paru, notamment, dans Le Populaire du 7 septembre 1936.

2. Archives départementales de l'Hérault (ADH), Journal Officiel. Débats parlementaires. Chambre des députés. Deuxième séance du 14 mars 1939. Intervention de M. Albert Sarraut.

3. ADH 4M1795. Communiqué du ministre de l'Intérieur (29/01/39).

4. D. Wingeate Pike, " 500000 réfugiés déferlent sur la France ", Historia, Hors série 22, 1971, p. $180-188$. 
que le décret-loi du 12 novembre 1938 légalisa l'internement administratif des " étrangers indésirables " dans des " camps spéciaux prévus à cet effet " et que, d'une manière générale, des mesures de plus en plus contraignantes vinrent amputer les droits de ceux qu'on appelait les "non-nationaux ». L'esprit de générosité du Front populaire céda la place à une " logique de police » qui avait pour but de s'assurer que la présence d'un étranger sur le territoire national ne constituait pas une menace pour l'ordre public ${ }^{5}$. Comme l'a très bien démontré Pierre Laborie, la société française de la fin des années trente fut marquée par une grave crise identitaire, dont le repli hexagonal, la xénophobie, l'antisémitisme, l'anticommunisme constituent les corollaires les plus immédiats ${ }^{6}$. Dans ce contexte troublé, on comprendra aisément que l'arrivée en masse des réfugiés espagnols de la guerre civile ait pu susciter des réactions de panique et de défiance, voire carrément de rejet.

Au même titre que les soldats, les prostituées, les nomades et les marginaux, les étrangers étaient considérés comme des groupes à risque, dans la mesure où ils pouvaient être porteurs de maladies contagieuses et infectieuses, et donc susceptibles de contaminer le corps sain de la population autochtone $e^{7}$. Afin d'assurer la "protection de la santé publique » et la "sauvegarde de la race ", le gouvernement renforça le contrôle sanitaire aux frontières terrestres et maritimes, par le biais notamment des campagnes de vaccination et des mesures de quarantaine pour les bateaux en provenance de l'étranger. Par la même occasion, il instaura le carnet sanitaire qui vint s'ajouter au certificat sanitaire déjà en vigueur dans la loi française. C'est la signification explicite du décret du 17 juin 1938 :

Art. 1 : Les étrangers appartenant à l'une des catégories pour lesquelles le certificat sanitaire est exigé aux termes de la réglementation actuelle ${ }^{8}$, devront être munis, en outre, d'un carnet sanitaire mentionnant les maladies dont ils sont atteints et les traitements auxquels ces maladies ont donné lieu. Seront également mentionnés sur le carnet sanitaire, les maladies que l'étranger contracterait ultérieurement ainsi que les traitements consécutifs à ces maladies.

Art. 2. Les parents, et d'une manière générale les personnes qui désirent rejoindre un travailleur étranger admis à séjourner en France devront, pour y être autorisées, obtenir le certificat sanitaire réglementaire ainsi que le carnet sanitaire prévu à l'article $1^{9}$.

5. J. Ponty, L'immigration dans les textes. France, 1789-2002, Paris, Belin, 2004 ; A. Sayad, "Immigration et pensée d'État ", Actes de la recherche en sciences sociales, 1999, vol. 129, n 1, p. 5-14.

6. P. Laborie, L'opinion française sous Vichy: les Français et la crise d'identité nationale 19361944, Paris, Seuil, 2001.

7. ADH 5M562. Contrôle sanitaire aux frontières (1932-1939).

8. ADH 2W614. Régime du séjour de l'étranger en France. Etaient exemptés du certificat sanitaire, les étrangers ayant résidé en France depuis plus de cinq ans, les femmes d'origine française ayant perdu leur nationalité à la suite d'un mariage avec un étranger, les étrangers ayant combattu pendant la guerre comme engagés volontaires ou comme légionnaires, les enfants de travailleurs étrangers âgés de moins de 18 ans et les étrangers au service d'agents diplomatiques ou consulaires régulièrement accrédités en France.

9. ADH. Journal Officiel, 19 juin 1938, p. 7521. 
Ainsi, le carnet sanitaire permettait-il d'effectuer le tri entre la partie saine et la partie malade de la population immigrée. Il indiquait si l'étranger était vacciné contre la variole, indemne de maladies mentales, d'épilepsie, de cécité, de surdité, de toxicomanies ${ }^{10}$, de maladies infectieuses ou parasitaires, de tuberculose pulmonaire, de maladies vénériennes ${ }^{11}$, de lèpre, de trachome ${ }^{12}$, ou de toute autre maladie chronique l'exposant à une hospitalisation prolongée. Dès lors, l'étranger non vacciné ou contagieux ne remplissait plus les conditions requises pour être admis sur le territoire français. En effet, celui-ci portait directement atteinte à l'ordre public, car il pouvait " affaiblir la race française » et être source de morbidité exceptionnelle. En outre, il se retrouvait à la charge de l'État, privait l'économie d'un travailleur et la défense nationale d'un soldat potentiel.

De façon générale, le gouvernement français mena une lutte acharnée contre les maladies infectieuses, parasitaires et vénériennes. Les instructions adressées aux préfets des départements par les ministres de l'Intérieur et de la Santé publique ne laissent planer aucun doute à ce sujet. Elles peuvent se résumer aux points suivants :

- la vaccination antivariolique, sauf dans les cas de cicatrices récentes;

- la vaccination antidiphtérique;

- le dépistage soigneux de tous les cas de maladies contagieuses;

- l'épouillage des porteurs de parasites et le traitement des galeux;

- la désinfection des personnes et des locaux ${ }^{13}$.

Indépendamment de la déclaration imposée aux médecins pour les maladies transmissibles ou épidémiques, les hôteliers et logeurs étaient tenus de signaler à la mairie tout cas de pathologie qui se manifesterait dans leur établissement. Dans la mesure du possible, les malades devaient être isolés pour qu'ils ne puissent pas propager la maladie. Jusqu'à la disparition complète de tout danger de contagion, on ne laisserait approcher des malades que les personnes appelées à les soigner, lesquelles prendraient toutes les précautions nécessaires pour empêcher la propagation du mal. Il était interdit de déverser des déjections -crachats, matières fécales, vomis, etc.- provenant d'un malade contagieux, sur le sol des voies publiques ou privées, des jardins, sur les fumiers et dans les cours d'eau. Ces déjections, recueillies dans des vases spéciaux, seraient enterrées profondément, mais seulement après avoir été désinfectées à la chaux vive. Pendant toute la durée d'une maladie infectieuse, les objets à usage personnel du malade et des

10. Par toxicomanies, les autorités françaises entendaient l'alcoolisme, l'opiomanie, la morphinomanie et la cocaïnomanie.

11. Au rang des principales maladies vénériennes, figuraient la syphilis, la blennorragie et le chancre mou.

12. Le trachome est une variété de conjonctivite contagieuse à l'origine de la première cause de cécité et qui se transmet par contact direct ou indirect, c'est-à-dire par les vêtements, les mains, des objets de toilette sales, entre autres. Les germes se transmettent également par l'intermédiaire des poussières et du sable transporté par le vent.

13. ADH 5M3. Réglementation, circulaires ministérielles, arrêtés préfectoraux (1935-1943); 5M565. Organisation de l'accueil et du contrôle des réfugiés en cas de guerre (1939-1940); 5M561. Contrôle sanitaire des réfugiés espagnols (1936-1940). 
personnes qui l'assistaient, de même que tous les objets contaminés ou souillés, seraient scrupuleusement désinfectés. Après la guérison ou le décès du malade, les lieux qu’il avait occupés devaient être lessivés et passés au grésyl ${ }^{14}$.

Si les autorités gouvernementales craignaient le contact entre population étrangère et population autochtone, elles redoutaient davantage encore celui des réfugiés en proie à la misère physique et mentale, à la promiscuité et à la malnutrition. Dans une circulaire datée du 24 août 1938, le ministre de la Santé publique rappelait au préfet de l'Hérault que les instructions prophylactiques données à maintes reprises par ses services étaient parfois perdues de vue, et que les divers services d'hygiène -Inspection départementale d'hygiène, Bureaux municipaux d'hygiène, Service sanitaire militaire, Service sanitaire maritimedevaient agir en liaison étroite afin de "préserver notre pays de tout danger d'importation de maladie contagieuse " :

Le moindre relâchement du contrôle sanitaire à l'égard des réfugiés de cette catégorie fait encourir à notre population des risques graves de contamination. En conséquence, j'ai l'honneur de vous rappeler que les réfugiés en provenance d'ESPAGNE [sic], quel que soit leur nombre, doivent être soumis à la visite médicale et à la vaccination ou revaccination antivariolique, l'épouillage et la désinfection étant, en outre, appliqués à ceux qui sont porteurs de parasites. Si l'isolement des malades s'impose sans préjudice des mesures complémentaires d'épouillage et de désinfection, il ne faut pas se dispenser d'exercer sur les contacts la surveillance sanitaire d'usage. Pour que je puisse me rendre compte de la stricte application des présentes instructions, je vous prie de me tenir informé à l'avenir :

$1^{\circ}$ ) de toute arrivée de réfugiés espagnols sur le territoire de votre département (liste nominative);

$2^{\circ}$ ) des mesures prophylactiques qui ont été prises à leur égard;

$3^{\circ}$ ) des incidents sanitaires survenus parmi eux;

$\left.4^{\circ}\right)$ des lieux ou des départements de destination qui leur ont été assignés ${ }^{15}$.

Plus d'un an après, dans le contexte de la Retirada, le même ministre demandait au préfet de l'Hérault, dans des termes sensiblement analogues, de redoubler de vigilance à l'égard de la variole et du typhus exanthématique qui frappaient de plein fouet certains réfugiés espagnols :

J'ai l'honneur, en ce qui me concerne, d'appeler à nouveau votre attention sur la nécessité de prendre vis-à-vis des réfugiés certaines mesures prophylactiques qui permettront de préserver nos populations de tout risque de contagion. Une vigilance toute particulière doit s'exercer à l'égard de la variole et du typhus exanthématique, maladies auxquelles sont exposées les personnes vivant en promiscuité et ayant eu à subir le froid et les privations.

Indépendamment de la visite médicale et de la vaccination antivariolique qui sont de rigueur, il faudra pratiquer l'épouillage et la désinsectisation du linge et des vêtements, chaque fois que la présence de parasites aura été constatée. Naturellement les malades et suspects devront être isolés et les contacts, soumis à la surveillance sanitaire ${ }^{16}$.

14. ADH 5M61. Organisation générale des services d'hygiène (1925-1939). Avec l'eau de Javel, le grésyl était un désinfectant bactéricide très efficace.

15. ADH 5M561. Ministre de la Santé publique Marc Rucart au préfet de l'Hérault (24/08/1938).

16. ADH 5M561. Ministre de la Santé publique Marc Rucart au préfet del'Hérault (28/01/1939). 
Comme ne manquait pas de le rappeler Albert Sarraut lors de la séance du 14 mars 1939 à l'Assemblée nationale, la question des réfugiés espagnols n'incombait pas uniquement au ministère de l'Intérieur mais intéressait de fait quatre départements. Le ministère de l'Intérieur veillait à la sécurité de l'ordre public, à l'hébergement et à la police des populations civiles réfugiées dans l'ensemble des départements. Le ministère de la Guerre s'occupait de l'organisation, du fonctionnement, de l'hygiène et de la discipline des « camps de concentration " aménagés dans le sud de la France. Le ministère de la Santé intervenait pour le contrôle des soins médicaux apportés aux réfugiés et " la protection de l'hygiène nationale contre certains effets de l'émigration espagnole ». Finalement, le ministère des Affaires étrangères menait des négociations diplomatiques concernant le rapatriement des réfugiés en Espagne et la participation des pays étrangers " au secours de cette masse humaine ". Bien entendu, les différents ministères ne travaillaient pas de façon isolée, mais en parfaite synergie sur cette question complexe :

Mais il va sans dire que cette apparente division du travail sur un même problème, ne signifie pas pour autant un système de cloisons étanches entre ces ministères selon lequel chacun ne s'occuperait que de ce qui regarde son compartiment. En fait, le caractère même et les aspects du problème des réfugiés imposent nécessairement la collaboration constante entre tous ces départements ministériels. La conversation, à cet égard, est quotidienne entre eux à cause de l'interdépendance des questions, et la coopération les fait solidaires dans toutes les solutions dont l'examen du problème fait apparaître la nécessité ${ }^{17}$.

\section{LA POLITIQUE SANITAIRE DU GOUVERNEMENT FRANÇAIS AVANT L'ARRIVÉE}

\section{DES MILICIENS ESPAGNOLS}

Avant le 5 février 1939, seuls les civils et les blessés pouvaient franchir la frontière franco-espagnole. Parmi les militaires, les blessés graves étaient admis à séjourner en France, tandis que les simples éclopés, les mutilés, les "blessés contaminés » et les blessés en voie de guérison étaient refoulés systématiquement en Espagne. Les blessés légers étaient soignés dans les centres sanitaires de Perpignan ${ }^{18}$, du Perthus, du Boulou, de Cerbère et de Prats-de-Mollo. Quant aux blessés graves, ils étaient conduits dans les hôpitaux de Perpignan, à savoir l'Hôpital Saint-Jean d'une capacité de 100 lits, l'ancien Hôpital militaire d'une capacité de 800 lits et l'ancien Hôpital Saint-Louis d'une capacité de 500 lits ${ }^{19}$. Afin de mieux répartir le poids de l'assistance et d'éviter la saturation des centres

17. ADH, Journal Officiel. Débats parlementaires. Chambre des députés. Deuxième séance du 14 mars 1939. Intervention de M. Albert Sarraut.

18. Infirmerie des anciens haras de Perpignan.

19. L'Hôpital Saint-Jean accueillait, de préférence, les malades contagieux, atteints de gale et de typhus. Les deux autres hôpitaux reprirent du service pour les besoins de la cause. Cf. S. Barba, De la frontière aux barbelés. Les chemins de la Retirada, Perpignan, Trabucaire, 2009, p. 145147. 
hospitaliers des Pyrénées-Orientales, on évacuait par trains sanitaires les réfugiés malades vers les hôpitaux des départements de l'intérieur.

Grâce au récit de la visite des ministres de l'Intérieur et de la Santé publique effectuée les 31 janvier et 1 février 1939 à Perpignan, il est possible de se faire une idée relativement précise de la politique sanitaire mise en oeuvre dans le département des Pyrénées-Orientales ${ }^{20}$.

Le 31 janvier au matin, Albert Sarraut et Marc Rucart débarquèrent en gare de Perpignan pour "se rendre compte sur place des dispositions prises pour appliquer les décisions du gouvernement tant en ce qui concerne la sécurité publique que les devoirs d'humanité et la protection des populations contre les dangers de la contamination $»^{21}$.

Dans un premier temps, les deux hommes visitèrent la localité du Boulou où les bains-douches municipaux avaient été transformés en centre d'accueil et en infirmerie. Sous la direction du docteur Bousquet, médecin-chef du centre sanitaire, et avec la participation active des infirmières de la Croix-Rouge de Perpignan, les réfugiés étaient ravitaillés et vaccinés, avant d'être dirigés en trains vers les départements de l'intérieur ${ }^{22}$. En compagnie du docteur Thomas, délégué du ministère de la Santé publique qui avait déjà dirigé les évacuations de Bilbao et de Santander, Marc Rucart inspecta les services sanitaires ainsi que le réfectoire installé dans la salle des fêtes de la municipalité.

Dans un deuxième temps, les ministres se rendirent au Perthus où ils furent reçus par le colonel Gauthier de la garde mobile. Dans les locaux de la mairie, une cantine et un centre de ravitaillement avaient été organisés par les soins d'un Comité de Quakers à l'intention des femmes, des enfants et des blessés de la guerre civile.

Du Perthus, ils se dirigèrent au fort de Bellegarde d'où l'on domine la plaine espagnole de l'Ampurdan et la ville de La Junquera. 2500 blessés, civils et militaires confondus, étaient entassés dans la forteresse transformée temporairement en infirmerie militaire ${ }^{23}$. De retour à Perpignan, ils en profitèrent pour inspecter les divers casernements occupés par les tirailleurs sénégalais et les gardes mobiles qui assuraient la surveillance des routes et le « ramassage des isolés » errant dans la montagne.

Le 1 février au matin, lors d'une conférence sanitaire technique qui réunissait le préfet Raoul Didkowski, le docteur Cavaillon, inspecteur de la Santé publique, le colonel Gauthier, commandant les forces de la frontière, le médecin-chef Thomas, délégué de la Santé publique et le marquis de Lillers, président de la

20. Cette visite a fait l'objet de très nombreux commentaires dans la presse nationale et locale, notamment dans Le Petit Méridional (organe républicain) et L'Éclair (organe conservateur) de Montpellier.

21. L'Éclair, 1/02/39.

22. Le Petit Méridional, 28/01/39. Aidé de la police, un groupe de dames de la Croix-Rouge de Perpignan, dirigea les enfants réfugiés vers l'établissement des bains-douches pour les vacciner. Après avoir été restaurés, ils furent conduits à Perpignan dans les locaux des anciens haras.

23. L'Éclair, 1/02/39. 4600 blessés furent également abrités dans les dépendances du fort de Salses. 
Croix-Rouge, Marc Rucart prit toute une série de mesures concrètes visant à mieux "améliorer et gérer l'exode ${ }^{24}$. Pour faire face au nombre insuffisant d'infirmières, une centaine de dames de la Croix-Rouge seraient réparties sur tous les "points névralgiques " de la frontière et sur tous les centres d'accueil du département. Trois membres parisiens du personnel sanitaire, placés sous la direction du médecin-chef adjoint du Service sanitaire maritime de Bordeaux, se rendraient prochainement à Perpignan pour organiser le service de santé et protéger en même temps la population française et les réfugiés espagnols contre des épidémies toujours à redouter. Les 4000 miliciens blessés qui se trouvaient au Boulou, au Perthus et à Prats-de-Mollo seraient transportés par trains sanitaires dans des hôpitaux situés le plus loin possible de la frontière afin de libérer des places dans les centres de Perpignan, Toulouse et la région. On établirait, enfin, un cordon sanitaire situé à la hauteur du cordon militaire, à une vingtaine de kilomètres en deçà de la frontière.

À l'issue de la conférence, MM. Sarraut et Rucart accompagnés du général Fagalde, commandant la $16^{\mathrm{e}}$ Région militaire, se mirent en route pour PortVendres, Banyuls et Cerbère, où la halle aux marchandises faisait office d'hôpital de campagne pour environ 1100 blessés :

Sur le quai de la gare un convoi de réfugiés composé de femmes et d'enfants attend le train qui va les emmener vers l'intérieur. Le sol de la gare a été arrosé de chlorure de chaux pour éviter toute contagion possible. Dans un coin des hardes sont entassées que l'on va brûler. Les deux ministres se dirigent vers la halle aux marchandises, où sont abrités 1100 blessés graves, mutilés, hâves, fiévreux. Un poste de pansements a été installé à l'entrée où médecins et infirmières travaillent sans relâche [...] Puis après s'être arrêtés devant une seconde salle où se trouvent des miliciens valides, les deux ministres remontent en voiture et gagnent Argelès-sur-Mer ${ }^{25}$.

La plupart de ces malheureux avaient été évacués des hôpitaux de Gérone et de Figueras, et avaient débarqué à Cerbère le 29 janvier à bord de deux cargos et d'un convoi de camions automobiles ${ }^{26}$. Avant de déjeuner à midi avec le préfet, les deux ministres firent un crochet par la plage d'Argelès-sur-Mer, où des miliciens encadrés par des gardes mobiles étaient en train de construire un « camp de concentration " destiné à abriter les futurs soldats de l'armée républicaine espagnole. Des baraques en bois étaient élevées dans la hâte et entourées de fil de fer barbelé.

De retour à Paris le 2 février au matin, MM. Sarraut et Rucart se félicitaient de la tâche accomplie par les autorités civiles et militaires, sans oublier de rendre hommage aux Catalans, dont certains firent preuve d'un remarquable courage et d'une exceptionnelle générosité. Voici ce qu'ils déclarèrent respectivement à la presse à leur descente du train en gare d'Orsay :

Albert Sarraut : "Je n'ai rien à ajouter aux déclarations que j'ai faites hier à Perpignan. La sécurité de la frontière est pleinement assurée. Il n’est pas question, pour

24. Le Petit Méridional, 2/02/39; L'Éclair, 2/02/39.

25. L'Éclair, 2/02/39, p. 2.

26. Le Petit Méridional, 30/01/39. 
le moment du moins, de prendre des mesures militaires, qui ne s'imposent nullement. On a seulement renforcé les effectifs de police afin d'empêcher l'exode non réglementé, des fugitifs" ".

Marc Rucart : " Je suis en mesure de vous assurer que les précautions sanitaires qui s'imposent ont été prises aussitôt que possible. En outre, je tiens à préciser que le dévouement de nos populations frontières est demeuré au-dessus de tout éloge. J’ai vu des maisons entièrement vidées de tout ce qui pouvait être utile à ces malheureux! ${ }^{27}$.

\section{LA POLITIQUE SANITAIRE DU GOUVERNEMENT FRANÇAIS APRÈS L'ARRIVÉE}

DES MILICIENS ESPAGNOLS

L'entrée en France des miliciens et des membres du gouvernement républicain, à compter du 5 février 1939, s'accompagna d'un renforcement du système de surveillance de la frontière et d'une réorganisation des services sanitaires. C'est ainsi que le département des Pyrénées-Orientales fut divisé en cinq secteurs : Cerbère, le Perthus, Prats-de-Mollo, Bourg-Madame et la ville de Perpignan. Les quatre premiers secteurs étaient placés sous la direction du secrétaire général de la préfecture, des sous-préfets de Céret et de Prades, tandis que le cinquième secteur relevait directement de la compétence du préfet ${ }^{28}$. À l'instar des civils, les miliciens blessés furent envoyés dans des hôpitaux de l'intérieur afin d'éviter l'engorgement de la ville de Perpignan, «l'une des plus touchées par l'exode » aux dires du général Ménard ${ }^{29}$. Par ailleurs, les autorités françaises encouragèrent au maximum les rapatriements en Espagne, par Hendaye dans un premier temps et par Port-Bou dans un deuxième temps.

Le 5 février, une conférence réunit au Perthus le préfet des Pyrénées-Orientales, le général Fagalde, le lieutenant-colonel espagnol chargé de la surveillance de la frontière et les autorités de La Junquera - notamment le commandant Somosa-, afin de fixer les règles qu'il y avait lieu d'adopter à l'égard des miliciens. Dès leur entrée sur le territoire français, ceux-ci seraient désarmés, dirigés en colonnes sur Le Boulou et conduits à pied ou en camion au camp de concentration d'Argelèssur-Mer ${ }^{30}$.

Le 7 février, le docteur Thomas se rendit au Boulou et au Perthus pour y examiner la situation sanitaire. D’après lui, la santé des soldats était relativement satisfaisante, en dépit de la fatigue et de l'épuisement provoqués par les combats. Les postes-secours accueillaient les petits blessés et les éclopés, alors que les blessés de guerre étaient soignés dans les hôpitaux de Perpignan et d'Arles-sur-Tech. À la gare internationale de Latour-de-Carol, des médecins français et espagnols ainsi que des infirmières de la Croix-Rouge faisaient tout leur possible pour prodiguer

27. L'Éclair, 3/02/39, p. 3.

28. Le Petit Méridional, 4/02/39.

29. Deux exemples : le 11 février, deux trains sanitaires amenèrent à Toulouse 260 blessés qui avaient été hospitalisés dans des villes du département des Pyrénées-Orientales. Le 12 février, 350 miliciens, hospitalisés à l'ancien hôpital de Perpignan, furent évacués dans un train sanitaire vers le centre de la France.

30. L'Éclair, 6/02/39. 
des soins de première urgence et refaire les pansements ${ }^{31}$. Aucun cas de contagion cependant n'était à signaler parmi la population française, ce qui prouve bien que les déclarations alarmistes diffusées à maintes reprises dans la presse réactionnaire relevaient du pur phantasme ${ }^{32}$.

Le 8 février, le ministre de la Marine donna des instructions précises pour qu'un certain nombre de paquebots de la marine marchande soient utilisés comme navires-hôpitaux et mis à la disposition des services chargés d'accueillir les réfugiés ${ }^{33}$. Au total, quatre bâtiments furent équipés : deux à Port-Vendres - "Asni " et "Maréchal Lyautey "- et deux autres à Marseille - "Patria » et "Providence ${ }^{34}$. Ce même jour, une mission de la Croix-Rouge fut dépêchée à Perpignan. Elle visita les trois hôpitaux de la capitale roussillonnaise, les installations du Boulou et du fort de Bellegarde. Pour remplacer et renforcer le personnel médical surmené par une tâche écrasante, elle annonça l'arrivée imminente dans les Pyrénées-Orientales de nombreuses infirmières toulousaines.

Le 9 février, le ministre de la Santé publique, non accompagné cette fois du ministre de l'Intérieur, se rendit à nouveau dans les Pyrénées-Orientales ${ }^{35}$. Au cours de sa visite, il inspecta les installations sanitaires de Perpignan, les trois infirmeries de la gare-frontière de Cerbère et le camp d'Argelès-sur-Mer. Afin de "décongestionner " celui-ci, il envisagea la création de deux nouveaux camps : l'un à Saint-Cyprien et l'autre au Barcarès, le long du littoral roussillonnais. Deux jours après, il se trouvait à Marseille pour définir avec les autorités locales les mesures sanitaires et sécuritaires à prendre à bord des navires-hôpitaux « Patria " et « Providence " ${ }^{36}$.

Si à la mi-février 1939 la politique sanitaire mise en place par le gouvernement français à l'égard des populations civiles s'avéra globalement efficace, en revanche, la situation dans les camps de concentration prenait une tournure de plus en plus dramatique.

Un rapport présenté le 18 février à l'Assemblée nationale par une délégation socialiste qui avait effectué une visite dans les Pyrénées-Orientales entre le 9 et le 14 février, jetait un éclairage très cru sur la situation sanitaire des miliciens espagnols. Au camp d'Argelès-sur-Mer, par exemple, le nombre des malades śélevait à 9000 , c'est-à-dire $12 \%$, et il n’y avait que 18 médecins. À SaintCyprien, il atteignait 15000 , soit $30 \%$, et là encore le personnel médical était

31. S. Barba, De la frontière aux barbelés, op cit., p. 102-103.

32. L'Éclair, 2/02/39, p. 2 : "Attention à la gale et autres maladies contagieuses! Parmi les réfugiés sur tous les points de la frontière, il y a de nombreux galeux. On les groupe tant bien que mal, en dehors des autres fugitifs. Signalons enfin que d'autres contagieux sont arrivés à la Preste ".

33. Le Petit Méridional, 9/02/39. Le 8 février, une cinquantaine de fonctionnaires espagnols du ministère des Affaires étrangères furent dirigés par train sanitaire sur Bordeaux et conduits ensuite à Bassens à bord du navire "La Habana » transformé pour l'occasion en navire-hôpital.

34. Le " Maréchal Lyautey " pouvait accueillir 600 blessés et l'« Asni " pouvait en recevoir 2000. Ce dernier était équipé de plusieurs salles d'opération.

35. Le Petit Méridional, 10/02/39.

36. L'Éclair, 12/02/39. 
nettement insuffisant ${ }^{37}$. Si le camp du Barcarès offrait des conditions de vie légèrement meilleures, la prolifération des mouches, des rats, des puces et des poux favorisait l'apparition de maladies épidémiques et infectieuses. Partout, le manque d'eau potable, l'absence de latrines et la précarité de l'hygiène, eurent des conséquences néfastes sur la santé des internés. Ceux qui parvinrent à échapper à la mort ${ }^{38}$, furent atteints de dysenterie, de pneumonie, de typhoïde, de tuberculose, d'avitaminose, de paludisme, voire même de lèpre! Le 16 mars, le député communiste Raymond Guyot interpella le gouvernement sur cette question lors d'une séance à l'Assemblée nationale. Voici le sombre portrait qu'il brossait de la situation dans les camps de concentration :

Il y avait, dans les camps, 30000 blessés et malades dont 3000 seulement ont été hospitalisés à Perpignan. Pourquoi le transfert des blessés et malades dans les hôpitaux d'autres départements n'est-il pas achevé? Dans les camps eux-mêmes, le service sanitaire est encore plus insuffisant. D’après le rapport de M. le médecin général Pélorquin, qui a tiré des conclusions fort intéressantes d'un voyage qu'il a mené dans les PyrénéesOrientales, on constate qu'à Argelès, où il y a 65000 réfugiés, il ne se trouve qu'un médecin-capitaine et quelques médecins-lieutenants, disposant de tentes sanitaires permettant d'hospitaliser au total 150 malades. Les réfugiés meurent parce qu'on ne peut les hospitaliser faute de place. Nous ne voulons pas que la honte en retombe sur le corps médical, ni que l'honneur de la France soit entaché. Par l'évacuation dans les hôpitaux, non seulement des Pyrénées-Orientales, mais des autres départements, par l'amélioration radicale de la situation des réfugiés, tant en ce qui concerne la nourriture et l'hygiène qu'en ce qui concerne les mesures sanitaires, par la désinfection des camps, des routes et des chemins dans les Pyrénées-Orientales et cela sous la responsabilité du service départemental d'hygiène, il faut venir au secours des blessés et des malades espagnols et éviter les épidémies meurtrières pour nos populations ${ }^{39}$.

Pour échapper à leur triste sort, nombre de miliciens n'hésitèrent pas à opter pour le retour en Espagne, la « réémigration » à destination de l'Amérique Latine et de l'URSS, l'incorporation dans les Compagnies de travailleurs étrangers, l'engagement dans les Régiments de marche de volontaires étrangers et dans la Légion étrangère.

Divers organismes nationaux et internationaux, au rang desquels le Secours populaire français, le Comité national catholique de secours aux enfants de Catalogne, le Comité basque d'aide aux réfugiés, le Comité des femmes socialistes suisses, le Comité national britannique, la Croix-Rouge belge, l'Union des femmes de France, le Comité international de la Croix-Rouge, etc., jouèrent en cette occasion un rôle d'auxiliaire des pouvoirs publics qui ne pouvaient financer seuls tous les frais d'assistance. C'est ainsi que le 5 février, le marquis de

37. D. Wingeate Pike, « 500000 réfugiés déferlent sur la France», op. cit., p. 183-184.

38. ADH, Journal Officiel. Débats parlementaires. Chambre des députés. Deuxième séance du 14 mars 1939. Interpellation de M. Raymond Guyot. Ce député livrait les informations suivantes : au seul cimetière de Perpignan, à la date du 23 février, reposaient 112 réfugiés dont 11 enfants inhumés. Au cours du mois de février, 200 décès auraient été enregistrés au vieil hôpital de Perpignan. 48 réfugiés seraient décédés dans le canton de Céret entre le 10 et le 17 février.

39. ADH, Journal Officiel. Débats parlementaires. Chambre des députés. Deuxième séance du 14 mars 1939. Interpellation de M. Raymond Guyot. 
Lillers rendait hommage à la Croix-Rouge française et au dévouement de tous les personnels qui avaient contribué à soulager la misère des réfugiés à la frontière des Pyrénées-Orientales :

Comme président de la Croix-Rouge française, je reviens extrêmement impressionné $\mathrm{du}$ voyage que je viens d'accomplir sur la frontière franco-espagnole avec le ministre de l'Intérieur et le ministre de la Santé publique qui avaient bien voulu me demander de les accompagner. J'ai pu constater sur place parmi les réfugiés espagnols, une misère poignante, atteignant en particulier des populations civiles : malades, vieillards, femmes et enfants. J'ai été frappé de la rapidité et de l'efficacité des mesures prises par les autorités françaises à tous les échelons de la hiérarchie pour assurer la protection sanitaire du pays. Une fois de plus la Croix-Rouge française a joué son rôle traditionnel d'auxiliaire des pouvoirs publics. Ses équipes d'infirmières, recrutées sur place et dont la plupart parlent la langue du pays, sont intervenues sous la direction des autorités compétentes, pour soulager toutes les misères physiques et morales des réfugiés. A Perpignan, à Cerbère, à Port-Vendres, au Perthus, à Prats-de-Mollo, au Boulou, pour ne citer que ces localités, les infirmières de la Croix-Rouge ont aidé à assurer le ravitaillement, ont donné les soins nécessaires et contribué à améliorer le sort des milliers de malheureux qui ont pu ainsi, dès leur arrivée sur le sol français, apprécier l'accueil de la France toujours humaine ${ }^{40}$.

Au terme de ce bref parcours, il apparait que la politique sanitaire menée par le gouvernement français à l'égard des réfugiés espagnols de la guerre civile ressortissait davantage à une " logique de police » qu'à une action humanitaire et généreuse. Au nom de la sûreté nationale et du droit d'asile, il incombait à l'État de préserver les populations françaises du risque de contagion, et de secourir et de sauver des êtres humains menacés de mort. Cela dit, la logique de police du gouvernement n'excluait nullement la logique humanitaire qui s'effectuait par le biais de l'aide privée nationale et internationale, notamment celle de la CroixRouge et du Secours populaire.

Dans la pratique, c'est le département des Pyrénées-Orientales qui accueillit la plupart des réfugiés malades et blessés de l'exode. À leur arrivée en France, ceuxci étaient vaccinés contre la variole et la diphtérie, épouillés et désinfectés. Sans aucun doute, les femmes et les enfants furent mieux traités que la population masculine, entrée en France à partir du 5 février et parquée dans les camps de concentration d'Argelès, de Saint-Cyprien et du Barcarès, où les conditions d'hygiène étaient absolument déplorables. Sous la houlette du préfet Raoul Didkowski, les autorités sanitaires civiles, militaires et maritimes, mirent en place un véritable réseau sanitaire composé de postes-secours (Cerbère, Perthus, Boulou, Prats-de-Mollo, Fort de Bellegarde), d'hôpitaux (Hôpital d'Arles-surTech, Hôpital Saint-Louis, Hôpital Saint-Jean et Hôpital de la Miséricorde de Perpignan) et de navires-hôpitaux ("Asni " et " Maréchal-Lyautey " amarrés à Port-Vendres). En raison de l'insuffisance de ces installations, les malades et les blessés durent être évacués par trains sanitaires vers les hôpitaux des régions limitrophes et des départements de l'intérieur de la France. 


\section{Annexe 1 : Circulaire $n^{\circ} 29$ du ministre de la Santé publique à l'attention du préfet de l'Hérault (3 mars 1939) (ADH 4M1799)}

J'ai l'honneur de vous adresser, ci-dessous, les instructions destinées à fixer ou à régulariser les conditions de gestion des hôpitaux auxiliaires qui ont recueilli des blessés ou malades, étrangers réfugiés d'Espagne et à déterminer les modalités de remboursement des frais occasionnés par leur hospitalisation, tant dans ces formations auxiliaires que dans les hôpitaux publics.

\section{I - HôpITAUX AUXILIAIRES \\ A-Gestion des Établissements}

Le gestionnaire qui est chargé de l'administration de ces établissements doit tenir :

-un registre des entrées et sorties des hospitalisés, mentionnant notamment leur identité, le diagnostic sommaire dont ils ont été l'objet, et les dates de l'arrivée et du départ de chacun d'eux;

-un registre des décès;

-un état nominatif, par catégorie, du personnel administratif, médical, infirmier et servant, indiquant le domicile de chaque argent, son emploi, les dates de prise et de cessation de fonctions, le tarif journalier de la rémunération, le nombre de journées de service;

-un livre-journal faisant ressortir la perception, l'emmagasinement et la conservation des denrées et objets mobiliers de toute nature, ainsi que leur distribution, un carnet spécial étant réservé pour l'essence et l'huile ; les consommations d'essence et d'huile, ainsi que les réparations et fournitures courantes éventuelles des voitures automobiles, après avoir été notées sur le carnet spécial, sont reportées tous les dix jours sur le livre-journal.

Le gestionnaire doit avoir à sa disposition un carnet de bons de commandes à souches qui est utilisé également pour les achats d'essence et d'huile;

Il dresse l'inventaire du matériel par catégories d'objets; il tient, en vue de régler directement les dépenses courantes d'exploitation, un carnet des avances qui pourront lui être accordées à concurrence d'un maximum de 10.000 frs après que vous l'aurez habilité auprès du Trésorier-Payeur Général ; ces avances sont renouvelables sur justifications, dans les conditions prévues par l'article 94 du décret du 31 mai 1862.

Les fournitures de pharmacie sont délivrées par l'hôpital public le plus proche de la formation auxiliaire.

B-Rétribution du Personnel

La rétribution du personnel est calculée, par journée ou par demi-journée de travail, sur les bases indiquées dans le tableau ci-joint (Annexe I).

\section{C-Réglement des dépenses}

Il faut distinguer les dépenses de personnel et les dépenses de matériel. Les premières sont mandatées par vos soins, suivant les règles de la comptabilité publique, sur production d'un état nominatif (annexe II) établi pour chaque 
formation et faisant ressortir les emplois des agents, le nombre des journées de service, les bases de leur rémunération et le montant de celle-ci ; cet état, est établi, mensuellement de préférence, par le gestionnaire ou le chef de service et arrêté par vous-même.

Les frais de transport du personnel civil n'appartenant pasà une administration publique sont remboursés dans les conditions fixées par l'annexe III.

Le règlement des frais analogues exposés par les fonctionnaires a lieu suivant les régles prévues à l'annexe IV.

Les dépenses de matériel sont réglées dans les conditions habituelles, que ces dépenses aient été effectuées par le gestionnaire ou qu'elles aient fait l'objet de mémoires de fournisseurs.

\section{II - HôpITAuX PUbLICS}

Les instructions antérieures qui mettaient à la charge de l'Assistance Médicale gratuite les dépenses des malades et blessés, réfugiés civils espagnols ont été modifiés par la circulaire $\mathrm{N}^{\circ} 2.245$, en date du 21 février 1939 de M. le Ministre de l'Intérieur. A dater du $1^{\text {er }}$ janvier 1929, ces dépenses comme celles qui résultent de l'hospitalisation des miliciens, quelle que soit leur nationalité d'origine, seront remboursées sur les crédits de l'Assistance aux réfugiés espagnols.

Les dépenses occasionnées par l'hospitalisation des blessés et malades dans les hôpitaux publics seront calculées sur la base du prix de journée applicable aux bénéficiaires de l'Assistance Médicale Gratuite ; en vue du règlement de ces dépenses, vous me transmettrez, à la fin de chaque mois, l'état des journées d'hospitalisation des blessés et malades de cette catégorie, établi conformément à l'Etat modèbe $\mathrm{B}$ annexe $\mathrm{N}^{\circ} 14$ de la circulaire du 16 mars 1937 ;

Les dépenses analogues auxquelles donnent lieu les formations annexes feront l'objet, dans les écritures de l'établissement principal, d'un compte horsbudget indiquant, par catégorie, les dépenses nécessitées par le fonctionnement de chaque formation (personnel, matériel, médicaments, alimentation...) ; celles-ci sont réglées sur production des états justificatifs prévus ci-dessus pour les hôpitaux auxiliaires.

Rentreront également dans le compte hors-budget les fournitures pharmaceutiques intéressant les formations annexes, et celles qui sont délivrées par les hôpitaux publics aux établissements auxiliaires sur le vu des bons de commande mentionnés ci-dessus.

\section{III - JUSTIFICATIONS DE L'EMPLOI DES CRÉDITS DÉLÉGUÉS}

Dans les 5 premiers jours de chaque mois, vous madresserez un état récapitulatif des mandats émis par vos soins au cours du mois précédent, sur les crédits qui vous auront été délégués.

Cet état sera accompagné, pour chaque mandat émis : de copies certifiées des états émargés, s’il s'agit de dépenses de personnel ; pour les dépenses de matériel, d'un relevé des mémoires ou factures et, pour celles de ces dépenses qui ont 
été réglées sur avances, d'une situation sommaire des justificatifs d'emploi des avances faites au gestionnaire, cette dernière pièce devant être vérifiée et visée par le Trésorier Payeur Général.

\section{LE MINISTRE Marc RUCART}

\section{Annexe 2 : Délégué sanitaire de la $\mathbf{I}^{\mathrm{re}}$ Circonscription de Montpellier à Monsieur le Préfet de l'Hérault (24/02/39) (ADH 5M561)}

J'ai l'honneur de vous rendre compte que les Services cliniques de l'Hôpital St Eloi confirment ce jour que la jeune ALONZO Marguerite, 6 ans, réfugiée Espagnole du camp de Villodève, est atteinte de fièvre typhoïde.

Un autre Espagnol SANCHEZ Pedro 31 ans a été hospitalisé le 16 février à l'Hôpital Pasteur, son homoculture est aussi positive.

Un troisième, SALARA Francisco est entré dans le même Etablissement le 15 de ce mois et attend la confirmation du même diagnostic.

Ces deux malades n'appartiennent pas au camp de Villodève. Les renseignements d'origine sont particulièrement difficiles à obtenir. J'ai chargé mon collègue, M. le Docteur GIRAUD, d'enquêter à ce sujet.

En ce qui concerne mes attributions cette déclaration a motivé une action immédiate de mes services dans le Camp.

L'emplacement occupé par l'enfant et toute la travée attenante a été débarrassée, la paille brûlée, le sol raclé, lavé au grésyl et recouvert de chlorure de chaux.

Or bon nombre de réfugiés déféquaient sur place et cachaient leurs déjections en les recouvrant de paille. Le récipient spécial qui avait été prêté à la mère de l'enfant alors qu'elle était en observation, a été retrouvé intact et n'a jamais été utilisé. Comme d'autre part, grands et petits circulaient en tous sens et notamment au niveau de la place occupée par l'enfant en fin de travée, il se peut que des germes aient été disséminés.

Enfin la maladie ayant évolué en période d'incubation dans le pays d'origine et dans le train, il se peut que d'autres cas surgissent inopinément.

J'ai donc prévenu immédiatement le Service Départemental d'Hygiène et $\mathrm{M}$. le Commissaire spécial de sorte que chacun, en ce qui le concerne, prévienne les centres d'hébergement qui ont recueilli nos réfugiés, d'avoir à redoubler de vigilance :

-Dans la surveillance de tous les cas fébriles qui surviendraient,

-Dans la désinfection systématique des selles.

L'installation au Camp de Villodève de W.C branchés sur l'égout et désinfectés tous les jours et plusieurs fois par jour par nettoyage à la lance et aspersion de chlorure de chaux, aura sans doute permis de limiter le dégât.

Mais la présence constatée de déjections dans la paille prouve que ces mesures étaient insuffisantes et la surveillance doit être exercée sur ce point particulier dans les centres nouvellement créés. MM. les Médecins-Inspecteurs des Secteurs 
devront être alertés et prévoir les mesures de défense ordinaire (organisation de la surveillance spéciale, avec consignes rigoureuses, désinfection des selles de tous les réfugiés; en cas de maladie recherche éventuelle des porteurs de germes, contrôle de la vaccination antérieure antityphoïdique et enfin vaccination massive si la situation s'aggravait).

\section{Docteur PAPPAS}

\section{Annexe 3 : Docteur VIGOUROUX à Monsieur le Préfet de l'Hérault (9/02/39) (ADH 4M1799)}

J'ai l'honneur, au moment où de nombreux réfugiés blessés ou malades espagnols sont susceptibles d'être reçus à Montpellier, de venir vous offrir le concours bénévole de mes services professionnels si vous le jugiez nécessaire :

$1^{\circ}$ Soit en vue d'assurer un service de consultation dans un centre d'hébergement ;

$2^{\circ}$ Soit en vue de donner des soins à des malades ou blessés.

J'ai pensé nécessaire d'offrir mes services à votre administration dans le cas où elle en aurait besoin, en même temps que coopérer à la loi de l'hospitalité française sans distinction d'opinion ou de croyances.

Agréez, Monsieur le Préfet, l'expression de mes sentiments respectueux. 